\title{
BMJ Open Self-reported health and smoking status, and body mass index: a case- control comparison based on GEN SCRIP (GENetics of SChizophRenia In Pakistan) data
}

Muhammad Ayub (i) , ${ }^{1}$ Arsalan Arsalan, ${ }^{2}$ Shams-ud-Din Ahmad Khan, ${ }^{3}$ Saqib Bajwa, ${ }^{4}$ Fahad Hussain, ${ }^{5}$ Muhammad Umar, ${ }^{5}$ Bakht Khizar, ${ }^{5}$ Muhammad Sibtain, ${ }^{5}$ Ayesha Butt, ${ }^{5}$ Mian Mukhtar-Ul-Haq, ${ }^{6}$ Imtiaz Ahmad Dogar, ${ }^{7}$ Moin Ahmad Ansari, ${ }^{8}$ Sadia Shafiq, ${ }^{9}$ Muhammad Tariq, ${ }^{9}$ Mian Iftikhar Hussain, ${ }^{10}$ Amina Nasar, ${ }^{1}$ Ali Burhan Mustafa, ${ }^{11}$ Rizwan Taj, ${ }^{12}$ Raza Ur Rehman, ${ }^{13}$ Atir Hanif Rajput, ${ }^{8}$ Syeda Ambreen, ${ }^{8}$ Syed Qalb-e-Hyder Naqvi, ${ }^{8}$ Khalid Mehmood, ${ }^{14}$ Muhammad Younis Khan, ${ }^{15}$ Jawad Ali, ${ }^{16}$ Nasir Mehmood, ${ }^{13}$ Ammara Amir, ${ }^{17}$ Tanveer Nasr, ${ }^{18}$ Fazal Rabbani, ${ }^{6}$ Adil Afridi, ${ }^{6}$ Zahid Nazar, ${ }^{6}$ Muhammad Idrees, ${ }^{19}$ Ahsan ul Haq Chishti, ${ }^{20}$ Rana Muzammil Shamsher Khan, ${ }^{21}$ Anisuzzaman Khan, ${ }^{22}$ Rubina Aslam, ${ }^{23}$ Muntazir Mehdi, ${ }^{24}$ Aftab Asif, ${ }^{25}$ Ali Zulqarnain, ${ }^{26}$ Jalil Afridi, ${ }^{9}$ Asif Hussain, ${ }^{9}$ Sibtain Anwar, ${ }^{27}$ Saad Salman, ${ }^{5}$ Inzemam Khan, ${ }^{5}$ Zia ul Mabood, ${ }^{5}$ Hamzalah Hamzalah, ${ }^{5}$ Adan Javed, ${ }^{5}$ Komal Nawaz, ${ }^{5}$ Kainat Zahra, ${ }^{5}$ Urooj Nayyar, ${ }^{5}$ Syeda Tooba, ${ }^{5}$ Ammara Ali Rajput, ${ }^{5}$ Anum Anjum, ${ }^{5}$ Ayesha Rehman, ${ }^{5}$ Maria Kanwal, ${ }^{5}$ Tahira Yasmeen, ${ }^{5}$ Sadia Hassan, ${ }^{5}$

To cite: Ayub M, Arsalan A, Khan S-DA, et al. Selfreported health and smoking status, and body mass index: a case-control comparison based on GEN SCRIP (GENetics of SChizophRenia In Pakistan) data. BMJ Open 2021;11:e042331. doi:10.1136/ bmjopen-2020-042331

- Prepublication history for this paper is available online. To view these files, please visit the journal online (http://dx.doi org/10.1136/bmjopen-2020042331).

Received 04 July 2020 Revised 11 December 2020 Accepted 17 March 2021

\section{Check for updates}

(c) Author(s) (or their employer(s)) 2021. Re-use permitted under CC BY-NC. No commercial re-use. See rights and permissions. Published by BMJ.

For numbered affiliations see end of article.

\section{Correspondence to} Dr Muhammad Ayub, Psychiatry, Queenn's University, Kingston, Canada; ma84@queensu.ca

Mariyam Ali Zaidi, ${ }^{5}$ Dur E Nayab, ${ }^{5}$ Muhammad Kamal, ${ }^{5}$ Bisma Jamil, ${ }^{5}$ Rida Malik, ${ }^{5}$ Intisham UI Haq, ${ }^{5}$ Zohra Bibi, ${ }^{5}$ Kalsoom Nawaz, ${ }^{5}$ Munaza Anwer, ${ }^{5}$ Afzal Javed, ${ }^{28}$ Nusrat Habib Rana, ${ }^{5}$ Muhammad Nasar Sayeed Khan, ${ }^{1}$ Farooq Naeem, ${ }^{29,30}$ Carlos N Pato, ${ }^{31}$ Michele T Pato, ${ }^{31}$ Saeed Farooq, ${ }^{32}$ James A Knowles ${ }^{33}$

\section{ABSTRACT}

Introduction Individuals with schizophrenia are at a high risk of physical health comorbidities and premature mortality. Cardiovascular and metabolic causes are an important contributor. There are gaps in monitoring, documenting and managing these physical health comorbidities. Because of their condition, patients themselves may not be aware of these comorbidities and may not be able to follow a lifestyle that prevents and manages the complications. In many lowincome and middle-income countries including Pakistan, the bulk of the burden of care for those struggling with schizophrenia falls on the families.

Objectives To determine the rate of self-reported physical health disorders and risk factors, like body mass index (BMI) and smoking, associated with cardiovascular and metabolic disorders in cases of schizophrenia compared with a group of mentally healthy controls.

Design A case-controlled, cross-sectional multicentre study of patients with schizophrenia in Pakistan.

Settings Multiple data collection sites across the country for patients, that is, public and private psychiatric OPDs (out patient departments), specialised psychiatric care facilities, and psychiatric wards of teaching and district level hospitals. Healthy controls were enrolled from the community.

\section{Strengths and limitations of this study}

It is a multicentre study, including individuals from different geographical locations, ethnicities and socioeconomic class, and uses a strict quality control mechanism for data collection.

It is, to our knowledge, the biggest study on physical health in schizophrenia in the Pakistani population.

- Non-random sampling technique.

- Results are based on subjective self-reports which can be affected by the variation in the pattern of reporting among individuals.

Participants We report a total of 6838 participants' data with (N 3411 (49.9\%)) cases of schizophrenia compared with a group of healthy controls (N $3427(50.1 \%)$ ). Results BMl (OR 0.98 ( $\mathrm{Cl} 0.97$ to 0.99$), p=0.0025)$, and the rate of smoking is higher in patients with schizophrenia than in controls. Problems with vision $(\mathrm{OR}$ 0.13 (0.08 to 0.2 ), joint pain (OR 0.18 (0.07 to 0.44$)$ ) and high cholesterol (OR 0.13 (0.05 to 0.35$)$ ) have higher reported prevalence in controls. The cases describe more physical health disorders in the category 'other' (OR 
4.65 (3.01 to 7.18)). This captures residual disorders not listed in the questionnaire.

Conclusions Participants with schizophrenia in comparison with controls report more disorders. The access in the 'other' category may be a reflection of undiagnosed disorders.

\section{INTRODUCTION}

Individuals suffering from schizophrenia are at an increased risk of developing comorbid non-psychiatric physical health disorders. This includes infections like tuberculosis, HIV, hepatitis B and C, ${ }^{12}$ osteoporosis, ${ }^{3}$ digestive system disorders, ${ }^{4}{ }^{5}$ respiratory system disorders, ${ }^{6-8}$ neurological diseases like movement disorders ${ }^{9}{ }^{10}$ and epilepsy. ${ }^{1112}$ A World Health Survey assessed the prevalence of nine different physical health disorders through a large cross-sectional general population study of 242952 individuals using random sampling from 48 low/middleincome countries (LMICs), including Pakistan. Selfreported disorders included arthritis, angina pectoris, asthma, diabetes, chronic back pain, visual impairment, hearing problems, edentulism and tuberculosis. Twenty-two hundred and twenty-four participants (1.1\%) reported that they had been diagnosed with schizophrenia/psychosis. The adjusted OR for individuals with psychosis was 4.05 (95\% CI 3.25 to 5.04) for multimorbidity defined as two or more of these disorders. ${ }^{13}$

Currently, the most widely studied physical health disorders are cardiovascular and metabolic disease. Several systematic reviews and meta-analyses show clear evidence of an increase in the risk of 1.4-2 times across all cardiovascular and metabolic diseases in individuals suffering from schizophrenia, ${ }^{14}$ resulting in significantly higher premature mortality. ${ }^{15}$ About $60 \%$ of the excess mortality in severe mental illness, is due to physical health disorders. ${ }^{16} 17$ The evidence from high-income countries shows that the mortality gap between schizophrenia and the general population is increasing, mainly due to cardiovascular disease. ${ }^{18}$ Evidence from LMICs also suggests that the average potential years of life lost for persons with severe mental illness is about 28 years. ${ }^{19}$ In a study from Ethiopia people with severe mental illness died 30 years prematurely and half of these deaths were because of infectious diseases. ${ }^{19}$

Excess morbidity and mortality are a result of some, potentially, modifiable risk factors including smoking, ${ }^{20}$ obesity ${ }^{21}$ and sedentary lifestyle. ${ }^{22}$ Lack of access to appropriate healthcare is another contributor. ${ }^{23}{ }^{24}$ Patients with severe mental illness are less likely to seek help for their physical health disorders ${ }^{2526}$ and the quality of care they receive is inferior to the people without mental illness. ${ }^{27-29}$ In individuals with schizophrenia, chronic diseases like diabetes mellitus, dyslipidaemia, hypertension are underdiagnosed and undertreated, ${ }^{30}$ blood pressure and cholesterol are less likely to be recorded by general practitioners (GPs). ${ }^{31}$ Consequences of mental illness like cognitive impairment, social isolation and suspiciousness may deter patients from seeking help. ${ }^{29}$ Individuals with schizophrenia have a higher body mass index (BMI) compared with the general population. The aetiology includes but is not limited to adverse effects of antipsychotics. Other factors involved are pretreatment/ premorbid genetic vulnerabilities, psychosocial and socioeconomic risk factors, and an unhealthy lifestyle. ${ }^{32}$

Most studies from well-developed healthcare systems have focused on cardiovascular diseases or metabolic disorders in individuals with schizophrenia. ${ }^{33-35}$ Individuals with psychosis are generally at high risk of multimorbidity, including physical health disorders, such as arthritis, chronic back pain, which may not increase the risk of premature mortality, but will have a significant negative impact on the quality of life. ${ }^{36}$ Despite the increased knowledge of heightened mortality from high-risk conditions such as diabetes, the research on multimorbidity (ie, two or more physical health comorbidities) in people with psychosis is still limited. The prevalence of and risk factors for developing disorders of physical health are well established, but the data from LMIC is sparse. ${ }^{14}$

Many studies of physical health disorders in schizophrenia have relied on self-reports. ${ }^{137-39}$ The prevalence of smoking has also been assessed with self-reports. ${ }^{40}$ In a US study, there was a consistency between the information from Medicaid, Medicare and primary data from selfreports, testifying to the validity of patient self-report. ${ }^{39} \mathrm{In}$ absence of electronic patient records and variation in the quality of case records, self-report is a viable approach to gathering information about physical health disorders in patients with schizophrenia in Pakistan.

This study is based on self-reported data on physical health disorders and smoking from a case-control study of individuals with schizophrenia. Additionally, we measured weight and height to assess the BMI. As part of a large, ongoing, multicentre, case-control study of the genetics of schizophrenia (GEN-SCRIP: GENetics of SChizophRenia In Pakistan), we have assessed comorbidity of different physical health disorders, BMI and history of smoking. We aim to compare the self-reported physical health status of participants with schizophrenia with those without this illness.

\section{METHODS}

Data presented here were generated from an ongoing, genetic case-control study initiated in 2018. The study aims to collect 10000 each of unrelated cases and controls from multiple sites in all the regions of Pakistan. Given the diversity of the population, we aimed to represent all the major racial groups in our data. Cases were recruited from clinics across sites in the country. The clinics were both public (ie, government institutions) and private sector. Clinicians referred previously diagnosed patients to the research team. Comparable controls were recruited from the local communities by advertising in educational institutions, vocational training centres, local malls. Study participants were asked to self-report physical health disorders and risk factors for cardiovascular and metabolic disease including smoking. We also measured the weight and height of the participants 


\begin{tabular}{llc}
\hline \multicolumn{2}{l}{ Table 1} & Summary of data collection sites by province \\
\hline Province & Cities & $\begin{array}{l}\text { Total } \\
\text { clinics }\end{array}$ \\
\hline $\begin{array}{l}\text { Khyber } \\
\text { Pakhtunkhwa }\end{array}$ & Dadar, Peshawar, Mardan & 7 \\
Punjab & $\begin{array}{l}\text { Faisalabad, Gujranwala, Khushab, } \\
\text { Sargodha, Lahore, Multan, }\end{array}$ & 13 \\
& $\begin{array}{l}\text { Sahiwal, Rahim Yar Khan, Sialkot } \\
\text { Sindh }\end{array}$ & Hyderabad, Karachi \\
Capital area & Islamabad & 3 \\
\hline
\end{tabular}

to calculate BMI. The data were collected between August 2018 and September 2019.

\section{Sites}

We collected data from 24 sites in the country. Data collection sites were spread across the country. Table 1 provides the provincial details of centres sampled.

Centres were spread across the country, included public sector hospitals and clinics and private sector clinics, to reach as many ethnic and racial groups as possible. Patients were recruited both from outpatients and inpatient departments.

\section{Interviewers}

Study interviewers had a graduate-level education in psychology or pharmacy. Interviewers received training in the administration of the study instruments (Screening Questionnaire and Diagnostic Interview for Psychosis and Affective Disorders (DI-PAD)). ${ }^{41}$ We selected these instruments to align with our collaborating team in the USA who have successfully employed these tools for over a decade and in order to make our data comparable to theirs. The questions for the Screening Questionnaire are from validated instruments and DI-PAD is based on questions from Diagnostic Interview for Genetic Studies (DIGS), ${ }^{42}$ a well-validated interview. Our senior diagnosticians were trained in New York City by our collaborating team. Senior diagnosticians who took part in initial training are three fully trained psychiatrists and one Ph.D. scholar. These individuals trained study interviewers. Interviews were supervised until inter-rater reliability was achieved, measured as Fleiss' kappa of 0.8 or more. Thus, training typically lasted approximately 4 days. We use the same stringent quality control system for data collection as used by our New York City collaborating team.

\section{Physical health assessment}

The physical health assessment consisted of the following three aspects.

1. BMI

Both for cases and controls we measured the height and weight and used that for calculation of BMI.

2. Assessments of physical health disorders

We used a Screening Questionnaire that has been developed for this purpose by our collaborating group, the Genomic Psychiatry Cohort Consortium in the USA. The consortium was established in 2008 and they have collected nearly 65000 participants for various studies. They have used the Screening Questionnaire in these studies. The Screening Questionnaire includes 32 questions and screens for mania, psychosis, depression, anxiety disorder, alcohol, nicotine and other substance use history. It is based on questions from well-validated interviews. The Screening Questionnaire also collects demographic information (ie, age, gender, and self-identifying race and ethnicity), medical conditions and disorders. The section on medical conditions starts with the question 'Have you ever been diagnosed with any of these medical conditions or disorders?' and then provides a list of disorders, for example, heart problems (examples: coronary heart disease, myocardial infarction), high cholesterol (hypercholesterolaemia). The participants can answer yes or no. The last question reads 'Any other medical or health problem. If yes, please describe:'

During the adaptation of the Screening Questionnaire, we used colloquial language for different disorders. For example, instead of diabetes, we used the word 'sugar' which is widely understood by laypeople.

3. Smoking status

The Screening Questionnaire has a section on smoking that we used for the collection of information about smoking.

\section{Psychiatric assessment}

For detailed psychopathological assessment of the cases, a semistructured interview was conducted using DI-PAD, that is, Diagnostic Interview for Psychosis and Affective Disorders-an 83 items interview schedule. It contains questions about psychopathology for psychotic and affective disturbances, premorbid adjustments of the patient, family history, treatment history and a section for interviewer ratings based on observations during the process. The screener and DI-PAD were developed by collaborators in the USA. ${ }^{41}$ The questions for the DI-PAD are from DIGS. $^{42}$ We translated and back translated the DI-PAD and the screener.

The process of translation was managed by a committee of bilingual researchers and linguists (AA, AN, MS, FN, FH, TN, MA). The instruments were in English and we translated them into Urdu. We initially commissioned three independent forward translations. These were reviewed by the committee and one consensus draft was provided to a separate group for back-translation. The back-translation was compared with the original draft and discrepancies identified were addressed through the second iteration of translation. The translation was then reviewed by five bilingual researchers in the field and their feedback was incorporated in the translated version. We then field tested the translated version in 50 participants. The feedback from interviewers was incorporated in the translation for further improvement. The final translated version was approved by the committee. 
The information was gathered through an interview with the patients, their accompanying caregiver when available, clinical records from the clinic where the patient was seen, and information from the treating clinical team.

\section{Participants and data collection}

Informed consent was taken from all participants. Patients fulfilling theDiagnostic and Statistical Manual of Mental Disorders Fifth Edition DSM-V criteria for schizophrenia or schizoaffective disorder depressed type were enrolled. Exclusion criteria for the cases were (1) poly-substance or any other abuse except cannabis before the onset of psychosis, (2) acute toxic psychosis, (3) patients with a history of severe head injury, (4) onset of schizophrenia after the age of 50, (5) neurological disorder before the onset of psychotic symptoms, (6) psychotic depression, (7) psychotic symptoms secondary to any medical conditions, (8) schizophrenia co-occurring with intellectual disability, (9) schizoaffective manic type. A record of the excluded patients was also maintained.

\section{Controls}

We aimed to enrol controls who did not have a personal or family history (in first degree relatives), of schizophrenia, major depression, obsessive-compulsive disorder, anxiety disorder, intellectual disability or bipolar disorder. We administered the Screening Questionnaire to these subjects. We used purposive sampling to recruit controls from matched communities. The controls had to be unrelated biologically to each other and the cases. We identified controls by advertising in educational institutions, professional training institutes and in the community. The lower limit for the age in controls was 23 .

\section{Quality control}

A stepwise standard operating procedure was designed and followed to make the process of enrollment uniform and alike among all sites. The data entered by interviewers were checked by a trainer/supervisor on daily basis and any issues were resolved by a face-to-face or telephone conversation. Ten per cent of patients from each centre were randomly selected and recalled for a repeat independent interview, by another trainer/supervisor. If a centre enrolled less than 100 patients, then we recalled a minimum of 10 patients for a second interview. If we identified any issues with an interviewer in a centre, we provided them additional training.

\section{Data analysis}

We used R V.3.6.1 (https://cran.r-project.org/bin/ windows/base/old/3.6.1/) for data analysis. For categorical variables, we provide percentages. We performed Fisher exact test or t-test as appropriate. We report frequencies of various physical health disorders. We performed logistic regression with case-control status as outcome measure and age and gender as covariates. We report ORs and $\mathrm{p}$ values for Wald's test. The other category in health issues covers anything not included in the list. We calculated BMI from height and weight. We used logistic regression with age and gender as covariates to examine the differences between cases and controls. In the screening questionnaire, there are four questions about smoking. We report the difference between cases and controls and use logistic regression with age and gender as covariates. We selected age and gender as covariates for these analyses because of prior evidence of their effect on physical health disorders, smoking and BMI.

\section{Patient or public involvement in study}

It was not appropriate or possible to involve patients or the public in the design, or conduct, or reporting, or dissemination plans of our research.

\section{RESULTS}

The schizophrenia cases are diagnosed using DSM-V criteria. The total number of cases was 3411 (49.9\%) and the total number of controls was 3427 (50.1\%). The mean age of cases was 33.50 (SD 10.0) years and of controls, it was 26.04 (SD 6.86) years.

\section{Participants}

There were $3411(49.9 \%)$ cases and $3427(50.1 \%)$ controls. Among cases, there were 2455 males and 956 females. In controls, 3032 were males and 395 females. The overall proportion of the females is lower than the males. The cases were older in age (mean 33.50 years (SD10.00)) than controls (mean 26.05 years (SD 6.84)) $\mathrm{p}$ value $<2.2 \mathrm{e}-16$.

\section{Self-reported physical health disorders}

Two hundred and eighty-seven $(8.38 \%)$ among controls reported one or more physical health disorders. This proportion was higher in cases $(n=516,15.11 \%)$. The adjusted OR for the difference was 1.26 (CI 1.06 to 1.5) with a $\mathrm{p}$ value of 0.01 .

Table 2 gives details of the 10 most commonly selfreported health problems. Case/Control status is the outcome measure. To control for multiple comparisons, alpha was set at 0.003 as the threshold for significance. The ORs are adjusted for age and gender. Cholesterol, joint pain and problems with vision were reported more frequently by controls. The numbers represent the participants who reported that they have been diagnosed with the condition, for example, for heart problems 12 participants in the case group and 8 participants in the control group said that they have been diagnosed with a heart condition. The ORs are adjusted for age and gender. Other problems (an umbrella term for any other problem) were higher in cases.

In figure 1, we show the percentage of self-reported physical health disorders in cases and controls. Apparent access of reported percentage in heart disease, high blood pressure, diabetes, digestive system disorders and 
Table 2 Comparison of self-reported health problems between cases and controls and results of logistic regression

\begin{tabular}{|c|c|c|c|c|}
\hline Disorders (N missing) & Cases N \% & Controls N \% & OR (Cl) Adjusted & Wald's p \\
\hline Heart disease (0) & $12(0.35)$ & $8(0.23)$ & 0.35 (0.12 to 0.99$)$ & 0.048 \\
\hline High BP (0) & $113(3.31)$ & $45(1.31)$ & 0.92 (0.6 to 1.41$)$ & 0.71 \\
\hline Liver disease (3) & $59(1.73)$ & $27(0.78)$ & 1.36 (0.8 to 2.31$)$ & 0.25 \\
\hline Sugar (diabetes) (1) & $84(2.46)$ & $33(0.96)$ & 0.93 (0.83 to 1.05$)$ & 0.24 \\
\hline Joint pain (3) & $14(0.41)$ & $18(0.52)$ & 0.18 (0.07 to 0.44$)$ & $0.00022^{\star}$ \\
\hline Epilepsy (1) & $11(0.32)$ & $3(0.08)$ & $3.96(1.01$ to 15.53$)$ & 0.048 \\
\hline Migraine (20) & $29(0.85)$ & $35(1.02)$ & 0.64 (0.37 to 1.11$)$ & 0.11 \\
\hline Visual problems (0) & $36(1.05)$ & $101(2.94)$ & 0.13 (0.08 to 0.2$)$ & $<2 \mathrm{e}-16^{\star}$ \\
\hline
\end{tabular}

${ }^{*}$ The significant $p$ values are in bold.

liver disease is not significant after adjustment for age and gender.

The BMI was significantly higher in patients with schizophrenia (mean 24.12 (SD 5.02)) compared with the control (23.53 mean (SD 3.96); OR 0.98 (CI 0.97 to $0.99), \mathrm{p}=0.00254)$.

In table 3, we provide the details of responses to questions about smoking. Smoking was more common in cases than in controls.

\section{DISCUSSION}

The major findings of the study are that people with schizophrenia had significantly higher BMIs and reported rates of smoking. The overall number of any physical health disorders was higher in cases. Many specific physical health disorders including cardiovascular system disorders did not differ significantly between those diagnosed with schizophrenia and the control population without a diagnosis of schizophrenia. The adjusted ORs of individuals reporting high cholesterol, visual problems and joint pains favoured controls. The only category that was higher in cases was 'other'. The question in the screening questionnaire about the 'other' category enquired about 'any other medical or health problem'. The 'other' category gives the participants a chance to describe any other physical health disorder that was not listed in the screening questionnaire, for example, heart problems (examples: coronary heart disease, myocardial infarction), high blood pressure (hypertension), and digestive problems (examples: gastrointestinal, celiac disease). As expected, response to this question resulted in a description of a large number of conditions in colloquial language that are difficult to summarise. We present this as one category as we suspect that this large number of 'other' represent unexplored and undiagnosed physical health disorders.

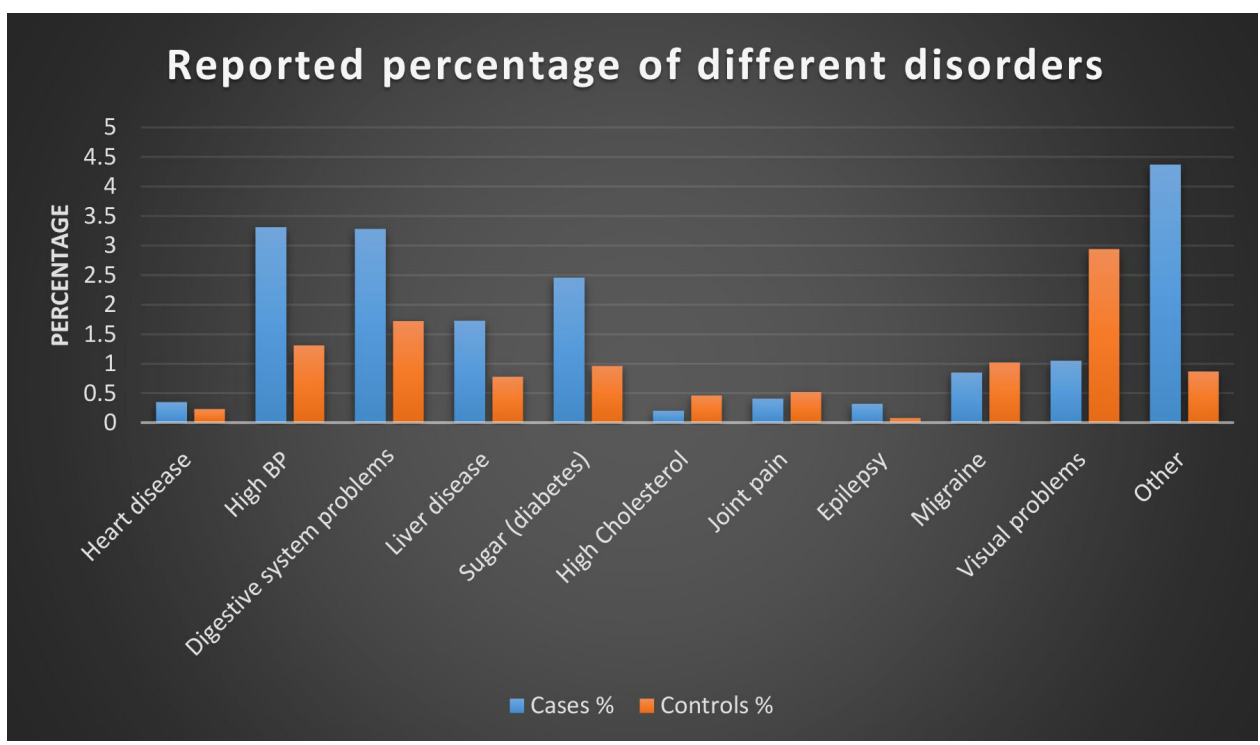

Figure 1 Percentage of self-reported physical health disorders in cases and controls. 
Table 3 Comparison of cases and controls for smoking

\begin{tabular}{lllll} 
Questions about smoking & Cases & Controls & OR (CI) & P value \\
\hline $\begin{array}{l}\text { Over your lifetime, have you smoked more } \\
\text { than } 100 \text { cigarettes? Include cigars, pipes and } \\
\text { chewing tobacco. }\end{array}$ & $1108 / 3410(32.49 \%)$ & $443 / 3425(12.93 \%)$ & $3.23(2.86$ to 3.67$)$ & $<2.2 \mathrm{e}-16$ \\
$\begin{array}{l}\text { Have you ever had a period of 1 month or more } \\
\text { when you smoked cigarettes every day? }\end{array}$ & $991 / 3413(29.03 \%)$ & $387 / 3424(11.30 \%)$ & $3.21(2.81$ to 3.66) & $<2.2 \mathrm{e}-16$ \\
$\begin{array}{l}\text { Did you usually smoke your first cigarette within } \\
\text { 1 hour after waking up? }\end{array}$ & $619 / 3407(18.16 \%)$ & $206 / 3425(6.01 \%)$ & $3.46(2.93$ to 4.11$)$ & $<2.2 \mathrm{e}-16$ \\
$\begin{array}{l}\text { Have you ever wanted to quit smoking or have } \\
\text { tried to quit smoking and found that you could } \\
\text { not? }\end{array}$ & $449 / 3408(13.17 \%)$ & $253 / 3424(7.38 \%)$ & $1.90(1.61$ to 2.24$)$ & $2.894 \mathrm{e}-15$ \\
\hline
\end{tabular}

Cl, 95\% Confidence Interval; OR, Odds Ratio.

\section{Metabolic syndrome}

The term metabolic syndrome has been used historically to describe a group of health conditions leading to increased risk of heart diseases and related problems. Physically, metabolic syndrome can manifest as increased waistline, high blood pressure, increased blood sugar, and obesity. The major risk factors for cardiometabolic disorders, that is, high BMI and cigarette smoking were present but surprisingly, disease conditions such as diabetes, high blood pressure and heart disease were not significantly higher in the individuals with schizophrenia compared with control. This is in contrast to almost universally reported findings of higher rates of cardiometabolic disorders in individuals with schizophrenia. ${ }^{14} 43$ High rates of metabolic syndrome are reported in those suffering from schizophrenia from India and Pakistan. In a systematic review of studies of metabolic syndrome from India, prevalence of metabolic syndrome in patients with schizophrenia was $29.83 \%$. The prevalence in community studies was $10.81 \%$ and in case-control studies, the OR of prevalence was 3.03 in cases compared with controls. ${ }^{44}$ In a hospital outpatient-based study from Pakistan with a sample size of fifty-six participants, $55.8 \%$ had metabolic syndrome. $^{45}$

There are a few possible explanations for this unusual finding including under-reporting by participants, and under-recognition of these conditions by healthcare providers. These explanations are not mutually exclusive. The most likely explanation is the lack of identification of cardiometabolic health problems in individuals with schizophrenia. Many studies in Europe and North America found that cardiovascular problems in individuals with schizophrenia are under-recognised, underrecorded and under-treated. ${ }^{24} 27303546$ Many barriers have been described that can lead to under-reporting of the symptoms in schizophrenia. Compared with healthy controls individuals with schizophrenia do not spontaneously report physical symptoms. ${ }^{47}$ Symptoms of schizophrenia, like cognitive impairment, suspiciousness, disorganisation and social isolation can result in lack of help-seeking or lack of compliance with care. When they seek help, lack of social skills and stigma of mental illness may pose additional barriers for them to receive appropriate care. ${ }^{48}$ The support for under-reporting and underrecognition of cardiometabolic symptoms comes from the finding that the 'other' category is reported more frequently in cases. This may be representing symptoms experienced by patients with schizophrenia that have not led to appropriate help-seeking, diagnosis and treatment.

In LMIC like Pakistan, patients with schizophrenia are primarily supported by their families. Almost all treatment costs are covered from out of pocket expenses. Economic reasons are an important factor in non-adherence to treatment for schizophrenia in Pakistan ${ }^{49} 50$ and neighbouring India with similar socioeconomic conditions. ${ }^{51-53}$ Physical healthcare may be less of a priority, especially if it is not causing acute symptoms. Patients are less likely to ask for assessment and treatment. Unless the physical health disorders are actively screened and managed, recognition of the metabolic syndrome and its components will remain low.

\section{Other conditions}

A study based on the World Health Survey described selfreported conditions from 48 LMICs including Pakistan. Nine somatic disorders were examined: arthritis, angina pectoris, asthma, diabetes, chronic back pain, visual impairment, hearing problems, edentulism and tuberculosis. Multimorbidity defined as two or more disorders had a significantly higher prevalence in individuals with subclinical psychosis, and psychosis. ${ }^{13}$ This is also consistent with the higher reported prevalence of these physical health disorders in other literature ${ }^{54-57}$ In the case of joint pains, the self-reported prevalence was higher in the control population, which may reflect a widely reported negative association between musculoskeletal conditions and schizophrenia. ${ }^{58}$ Additionally, in the case of painful musculoskeletal disorders, it has been postulated that people with schizophrenia may be less likely to present with painful conditions based on indirect evidence that they may experience hypoanalgesia. ${ }^{59}$

A lower rate of problems with vision has not been reported before in people with schizophrenia. There are studies to suggest that visual problems are positively 
associated with schizophrenia. ${ }^{54} 56$ The eye problems associated with schizophrenia include eye movement disorder $^{60}{ }^{6}$ strabismus $^{61-64}$ and visual impairment. ${ }^{65} 66$ Certain systemic infections can have eye manifestations and can lead to schizophrenia. These include toxoplasmosis $^{67}$ and rubella. ${ }^{68}$ The possible explanation for a higher reported rate in controls in this study is lack of awareness among schizophrenia patients about the deficits and so lack of help-seeking, investigation and treatment.

\section{Clinical implications}

Monitoring of physical health should be an integral part of the care for individuals with schizophrenia. This can be encouraged through clinical audit and training of healthcare providers.

\section{Research implications}

Further work that employs assessment of physical health by health professionals rather than self-report will provide a better estimate of comorbidity in patients with schizophrenia in Pakistan.

\section{Limitations}

The strengths of this study include a large sample size with a control group a uniform data collection procedures by trained assessors with acceptable interrater reliability from multiple sites and the use of a methodology and instruments that have been well established in previous research. ${ }^{41}$ However, the findings should be interpreted in light of a some important limitations. One limitation is that the data on physical health was self/family reported, without corroboration from other data sources (eg, medical records). It was not feasible to conduct full physical examinations for the number of multi-morbidities reported. We did, however, collect information from family members in addition to participant self-reports, which strengthens our data.

In part due to the diverse, potentially heterogenous nature of our sample, there may be a difference in reporting patterns between and within the cases and controls. However, many factors can influence the reporting of physical symptoms and physical disorders. For instance, because of cognitive deficits, individuals with schizophrenia may be unaware of physical symptoms $^{6970}$ and antipsychotics reduce the pain sensitivity ${ }^{6970}$ that would affect the reporting of physical health disorders. Additionally, a lack of social skills ${ }^{29}$ and difficulties in communicating physical needs also affect their reporting pattern. $^{71}$

Finally, the patients and controls were not selected randomly. For cases, everyone who met the criteria and consented was enrolled in the study. Due to the lack of services, severe and chronic cases are more likely to seek help from psychiatric facilities. This should result in higher levels of morbidity in our cases.

\section{Conclusions}

We report a large cross-sectional study that shows the selfreported physical health disorders in participants with schizophrenia. The overall rate of self reported physical health disorders was higher in schizophrenia mainly because they reported a higher number of problems in the 'other' category. Apart from a higher rate of increased cholesterol among controls, the cardiometabolic symptoms were not different between the two groups. However, the major risk factors such as BMI and smoking were found significantly higher in cases with schizophrenia. Prior evidence from the literature suggests that individuals with schizophrenia are less aware of their physical health disorders and less likely to seek healthcare. Excess of reported disorders in the 'other' category may reflect disorders that have not been reported to and investigated by the healthcare providers.

\section{Author affiliations}

${ }^{1}$ Psychiatry, Queen's University, Kingston, Ontario, Canada

${ }^{2}$ Pharmacy, University of Peshawar, Peshawar, Pakistan

${ }^{3}$ Psychiatry, Al-Shams Hospital Sargodha, Sargodha, Pakistan

${ }^{4}$ Psychiatry, District Headquarter Hospital Gujranwala, Gujranwala, Pakistan

${ }^{5}$ Research, Lahore Institute of Research and Development, Lahore, Pakistan

${ }^{6}$ Psychiatry, Lady Reading Hospital, Peshawar, Pakistan

${ }^{7}$ Department of Psychiatry, District Head Quarter Hospital, Faisalabad, Pakistan

${ }^{8}$ Psychiatry, Sir Cowasjee Jehangir Institute of Psychiatry, Hyderabad, Pakistan

${ }^{9}$ Psychiatry, Shafique Psychiatric Clinic, Peshawar, Pakistan

${ }^{10}$ Psychiatry, Mian Iftikhar Psychiatric Hospital, Peshawar, Pakistan

${ }^{11}$ Psychiatry, Sheikh Zayed Hospital Rahim Yar Khan, Rahim Yar Khan, Pakistan

${ }^{12}$ Psychiatry, Pakistan Institute of Medical Sciences, Islamabad, Pakistan

${ }^{13}$ Psychiatry and Psychology, Karwan-E-Hayat Psychiatric Hospital And

Rehabilitation Centre, Karachi, Pakistan

${ }^{14}$ Psychiatry, Ar-Rahma Hospital, Multan, Pakistan

${ }^{15}$ Psychiatry, Khushal Medical Centre, Peshawar, Pakistan

${ }^{16}$ Psychiatry, Government Mental and General Hospital Dadar, Mansehra, Pakistan

${ }^{17}$ Psychiatry, Sir Gangaraam Hospital, Lahore, Pakistan

${ }^{18}$ Psychology, Ameena Clinic, Gujranwala, Pakistan

${ }^{19}$ Idrees Private Clinic, Akbar Medical Centre, Peshawar, Pakistan

${ }^{20}$ Psychiatry, Recovery Rehab Centre, Lahore, Pakistan

${ }^{21}$ Psychiatry, Allama Iqbal Teaching Hospital Sialkot, Sialkot, Pakistan

${ }^{22}$ Psychiatry, Nai Zindage Psychiatric Hospital, Multan, Pakistan

${ }^{23}$ Psychiatry, Jinnah Hospital, Lahore, Pakistan

${ }^{24}$ Psychiatry, Haji Abdul Qayyum Hospital, Sahiwal, Pakistan

${ }^{25}$ Psychiatry, Mayo Hospital Lahore, Lahore, Pakistan

${ }^{26}$ Psychiatry, Aleeze Neuro Psychiatric Centre, Sargodha, Pakistan

${ }^{27}$ Psychiatry, Sibtain Anwar Psychiatric Hospital, Mardan, Pakistan

${ }^{28}$ Mental Health, Pakistan Psychiatric Research Centre, Fountain House, Lahore, Pakistan

${ }^{29}$ Psychiatry, University of Toronto Faculty of Medicine, Toronto, Ontario, Canada ${ }^{30}$ Psychiatry, Center for Addiction and Mental Health, Toronto, Ontario, Canada

${ }^{31}$ Behavioral Sciences, State University New York, Downstate Medical Centre,

Broklyn, New York, USA

${ }^{32}$ Psychiatry, Keele University, Keele, UK

${ }^{33}$ Cell Biology, State University New York, Downstate Medical Centre, Broklyn, New York, USA

\section{Twitter Muhammad Ayub @Ayub1985}

Contributors These authors designed the study: MA, AAr, SB, MS, AB, MNSK, FN, CNP, MTP and JK. These authors contributed to data collection: MA, AAr, S-u-DAK, SB, FH, MU, BK, MS, AB, MMUH, IAD, MAA, SSh, MT, MIH, AN, ABM, RT, RuR, AHR, SAm, SQ-e-HN, KM, MYK, JAI, NM, AAm, TN, FR, AAf, ZN, MI, AuHC, RMSK, AK, RA, MM, AAs, AZ, JAf, AHu, SAn, SSa, IK, ZuM, HH, AdJ, KoN, KZ, UN, ST, AAR, AAn, AR, MK, TY, SH, MAZ, DeN, MK, BJ, RM, IuH, ZB, KaN, MA, AfJ, NHR, MNSK, FN, SF and JAK. These authors contributed to data analysis and manuscript preparation: MA, AAr, FH, MU, BK, ZuM, FN, CNP, MTP, SF, and JAK. These authors edited and approved the manuscript: MA, AAr, S-u-DAK, SB, FH, MU, BK, MS, AB, MMuH, IAD, MAA, SSh, MT, MIH, AN, ABM, RT, RuR, AHR, SAm, SQ-e-HN, KM, MYK, JAl, NM, AAm, TN, FR, AAf, ZN, MI, AuHC, RMSK, AK, RA, MM, AAs, AZ, JAf, AHu, SAn, SSa, IK, 
Zia ul Mabood, HH, AdJ, KoN, KZ, UN, ST, AAR, AAn, AR, MK, TY, SH, MAZ, DeN, MK, BJ, RM, IuH, ZB, KaN, MA, AfJ, NHR, MNSK, FN, CNP, MTP, SF and JAK.

Funding This work was supported by the 'National Institute of Mental Health' (grant number: 1R01MH112904-01).

Competing interests None declared.

Patient and public involvement Patients and/or the public were not involved in the design, or conduct, or reporting, or dissemination plans of this research.

\section{Patient consent for publication Not required}

Ethics approval This study was conducted under the ethics approval from Institutional Research Ethics Board of teaching hospitals and the National Bioethics Committee, Islamabad, Pakistan, Reference Number. 4-87/NBC-367/19/2060.

Provenance and peer review Not commissioned; externally peer reviewed.

Data availability statement No data are available. This is an ongoing study and data are not available at this stage.

Open access This is an open access article distributed in accordance with the Creative Commons Attribution Non Commercial (CC BY-NC 4.0) license, which permits others to distribute, remix, adapt, build upon this work non-commercially, and license their derivative works on different terms, provided the original work is properly cited, appropriate credit is given, any changes made indicated, and the use is non-commercial. See: http://creativecommons.org/licenses/by-nc/4.0/.

\section{ORCID iD}

Muhammad Ayub http://orcid.org/0000-0002-7111-1571

\section{REFERENCES}

1 Bauer-Staeb C, Jörgensen L, Lewis G, et al. Prevalence and risk factors for HIV, hepatitis $B$, and hepatitis $C$ in people with severe mental illness: a total population study of Sweden. Lancet Psychiatry 2017;4:685-93.

2 Campos LN, Guimarães MDC, Carmo RA, et al. HIV, syphilis, and hepatitis $B$ and $C$ prevalence among patients with mental illness: a review of the literature. Cad Saude Publica 2008;24:s607-20.

3 Tseng P-T, Chen Y-W, Yeh P-Y, et al. Bone mineral density in schizophrenia: an update of current meta-analysis and literature review under guideline of PRISMA. Medicine 2015;94:e1967.

4 Severance EG, Prandovszky E, Castiglione J, et al. Gastroenterology issues in schizophrenia: why the gut matters. Curr Psychiatry Rep 2015;17:27.

5 Severance EG, Yolken RH, Eaton WW. Autoimmune diseases, gastrointestinal disorders and the microbiome in schizophrenia: more than a gut feeling. Schizophr Res 2016;176:23-35.

6 Chafetz L, White MC, Collins-Bride G, et al. The poor general health of the severely mentally ill: impact of schizophrenic diagnosis. Community Ment Health J 2005;41:169-84.

7 Himelhoch S, Lehman A, Kreyenbuhl J, et al. Prevalence of chronic obstructive pulmonary disease among those with serious mental illness. Am J Psychiatry 2004;161:2317-9.

8 Lawrence D, Kisely S, Pais J. The epidemiology of excess mortality in people with mental illness. Can J Psychiatry 2010;55:752-60.

9 Fenton WS. Prevalence of spontaneous dyskinesia in schizophrenia. J Clin Psychiatry 2000;61:10-14.

10 Pappa S, Dazzan P. Spontaneous movement disorders in antipsychotic-naive patients with first-episode psychoses: a systematic review. Psychol Med 2009;39:1065-76.

11 Fruchter E, Kapara O, Reichenberg A, et al. Longitudinal association between epilepsy and schizophrenia: a population-based study. Epilepsy Behav 2014;31:291-4

12 Taylor DC. Schizophrenias and epilepsies: why? when? how? Epilepsy Behav 2003;4:474-82.

13 Stubbs B, Koyanagi A, Veronese N, et al. Physical multimorbidity and psychosis: comprehensive cross sectional analysis including 242,952 people across 48 low- and middle-income countries. BMC Med 2016;14:189.

14 Firth J, Siddiqi N, Koyanagi A, et al. The lancet psychiatry commission: a blueprint for protecting physical health in people with mental illness. Lancet Psychiatry 2019;6:675-712.

15 Hjorthøj C, Stürup AE, McGrath JJ, et al. Years of potential life lost and life expectancy in schizophrenia: a systematic review and metaanalysis. Lancet Psychiatry 2017;4:295-301.

16 Parks J, Svendsen D, Singer P, eds. Morbidity and mortality in people with serious mental illness, 2006.
17 Vreeland B. Treatment decisions in major mental illness: weighing the outcomes. J Clin Psychiatry 2007;68:5-11.

18 Lawrence D, Hancock KJ, Kisely S. The gap in life expectancy from preventable physical illness in psychiatric patients in Western Australia: retrospective analysis of population based registers. BMJ 2013;346:f2539.

19 Fekadu A, Medhin G, Kebede D, et al. Excess mortality in severe mental illness: 10-year population-based cohort study in rural Ethiopia. Br J Psychiatry 2015;206:289-96.

20 Myles N, Newall HD, Curtis J, et al. Tobacco use before, at, and after first-episode psychosis: a systematic meta-analysis. J Clin Psychiatry 2012;73:468-75.

21 Bradshaw T, Mairs H. Obesity and serious mental ill health: a critical review of the literature. Healthcare 2014;2:166-82.

22 Mamakou V, Thanopoulou A, Gonidakis F, et al. Schizophrenia and type 2 diabetes mellitus. Psychiatriki 2018;29:64-73.

23 Fagiolini A, Goracci A. The effects of undertreated chronic medical illnesses in patients with severe mental disorders. J Clin Psychiatry 2009;70:22-9.

24 Mitchell AJ, Malone D, Doebbeling CC. Quality of medical care for people with and without comorbid mental illness and substance misuse: systematic review of comparative studies. $\mathrm{Br} J$ Psychiatry 2009;194:491-9.

25 Cradock-O'Leary J, Young AS, Yano EM, et al. Use of general medical services by Va patients with psychiatric disorders. Psychiatr Serv 2002;53:874-8.

26 Salsberry PJ, Chipps E, Kennedy C. Use of general medical services among Medicaid patients with severe and persistent mental illness. Psychiatr Serv 2005;56:458-62.

27 Kisely S, Campbell LA, Wang Y. Treatment of ischaemic heart disease and stroke in individuals with psychosis under universal healthcare. Br J Psychiatry 2009;195:545-50.

28 Kisely S, Smith M, Lawrence D, et al. Inequitable access for mentally ill patients to some medically necessary procedures. CMAJ 2007;176:779-84.

29 Phelan M, Stradins L, Morrison S. Physical health of people with severe mental illness. BMJ 2001;322:443-4.

30 Nasrallah HA, Meyer JM, Goff DC, et al. Low rates of treatment for hypertension, dyslipidemia and diabetes in schizophrenia: data from the CATIE schizophrenia trial sample at baseline. Schizophr Res 2006;86:15-22.

31 Roberts L, Roalfe A, Wilson S, et al. Physical health care of patients with schizophrenia in primary care: a comparative study. Fam Pract 2007;24:34-40.

32 Manu P, Dima L, Shulman M, et al. Weight gain and obesity in schizophrenia: epidemiology, pathobiology, and management. Acta Psychiatr Scand 2015;132:97-108.

33 Crump C, Winkleby MA, Sundquist K, et al. Comorbidities and mortality in persons with schizophrenia: a Swedish national cohort study. Am J Psychiatry 2013;170:324-33.

34 Mitchell AJ, Lord O, Malone D. Differences in the prescribing of medication for physical disorders in individuals with $\mathrm{V}$. without mental illness: meta-analysis. Br J Psychiatry 2012;201:435-43.

35 Smith DJ, Langan J, McLean G, et al. Schizophrenia is associated with excess multiple physical-health comorbidities but low levels of recorded cardiovascular disease in primary care: cross-sectional study. BMJ Open 2013;3 doi:10.1136/bmjopen-2013-002808

36 Stubbs B, Mitchell AJ, De Hert M, et al. The prevalence and moderators of clinical pain in people with schizophrenia: a systematic review and large scale meta-analysis. Schizophr Res 2014;160:1-8.

37 Baughman KR, Bonfine N, Dugan SE, et al. Disease burden among individuals with severe mental illness in a community setting. Community Ment Health J 2016;52:424-32.

38 Dickerson FB, Pater A, Origoni AE. Health behaviors and health status of older women with schizophrenia. Psychiatr Serv 2002;53:882-4

39 Dixon L, Weiden P, Delahanty J, et al. Prevalence and correlates of diabetes in national schizophrenia samples. Schizophr Bull 2000;26:903-12.

40 Lasser K, Boyd JW, Woolhandler S, et al. Smoking and mental illness: a population-based prevalence study. JAMA 2000;284:2606-10.

41 Pato MT, Sobell JL, Medeiros $\mathrm{H}$, et al. The genomic psychiatry cohort: partners in discovery. Am J Med Genet B Neuropsychiatr Genet 2013;162B:306-12.

42 Nurnberger Jl, Blehar MC, Kaufmann CA, et al. Diagnostic interview for genetic studies. rationale, unique features, and training. NIMH genetics initiative. Arch Gen Psychiatry 1994;51:849-59.

43 Vancampfort D, Stubbs B, Mitchell AJ, et al. Risk of metabolic syndrome and its components in people with schizophrenia and 
related psychotic disorders, bipolar disorder and major depressive disorder: a systematic review and meta-analysis. World Psychiatry 2015;14:339-47.

44 Ganesh S, Ashok AH, Kumar CN, et al. Prevalence and determinants of metabolic syndrome in patients with schizophrenia: a systematic review and meta-analysis of Indian studies. Asian J Psychiatr 2016;22:86-92.

45 Ullah A, Khan SM, Khan FH. Metabolic syndrome in patients with schizophrenia. J Liaquat Univ Med Health Sci 2015;14:63-7.

46 Osborn DPJ, King MB, Nazareth I. Participation in screening for cardiovascular risk by people with schizophrenia or similar mental illnesses: cross sectional study in general practice. $B M J$ 2003;326:1122-3.

47 Jeste DV, Gladsjo JA, Lindamer LA, et al. Medical comorbidity in schizophrenia. Schizophr Bull 1996;22:413-30.

48 Goldman LS. Medical illness in patients with schizophrenia. J Clin Psychiatry 1999;60:10-15.

49 Ahmad I, Khalily MT, Hallahan B. Reasons associated with treatment non-adherence in schizophrenia in a Pakistan cohort. Asian $J$ Psychiatr 2017;30:39-43.

50 Taj R, Khan S. A study of reasons of non-compliance to psychiatric treatment. J Ayub Med Coll Abbottabad 2005;17:26-8.

51 Chaudhari B, Saldanha D, Kadiani A, et al. Evaluation of treatment adherence in outpatients with schizophrenia. Ind Psychiatry J 2017;26:215-22.

52 Roy R, Jahan M, Kumari S, et al. Reasons for drug non-compliance of psychiatric patients: a centre based study. IJIAAP 2005;31:24-8.

53 Chandra IS, Kumar KL, Reddy MP, et al. Attitudes toward medication and reasons for non-compliance in patients with schizophrenia. Indian J Psychol Med 2014;36:294-8.

54 Anthony SA. Focus on eye care in schizophrenia. Clin Exp Optom 2019;102:385-93.

55 Clancy MJ, Clarke MC, Connor DJ, et al. The prevalence of psychosis in epilepsy; a systematic review and meta-analysis. BMC Psychiatry 2014;14:75

56 Silverstein SM, Rosen R. Schizophrenia and the eye. Schizophr Res Cogn 2015;2:46-55

57 Wotton CJ, Goldacre MJ. Coexistence of schizophrenia and epilepsy: record-linkage studies. Epilepsia 2012;53:e71-4.
58 Euesden J, Breen G, Farmer A, et al. The relationship between schizophrenia and rheumatoid arthritis revisited: genetic and epidemiological analyses. Am J Med Genet B Neuropsychiatr Genet 2015;168B:81-8.

59 Potvin S, Marchand S. Hypoalgesia in schizophrenia is independent of antipsychotic drugs: a systematic quantitative review of experimental studies. Pain 2008:138:70-8.

60 Rommelse NNJ, Van der Stigchel S, Sergeant JA. A review on eye movement studies in childhood and adolescent psychiatry. Brain Cogn 2008;68:391-414.

61 Hassan MB, Hodge DO, Mohney BG. Prevalence of mental health illness among patients with adult-onset strabismus. Strabismus 2015;23:105-10.

62 Leskowitz E. Strabismus and schizophrenia. Am J Psychiatry 1984;141:614.

63 Mohney BG, McKenzie JA, Capo JA, et al. Mental illness in young adults who had strabismus as children. Pediatrics 2008;122:1033-8.

64 Schiffman J, Maeda JA, Hayashi K, et al. Premorbid childhood ocular alignment abnormalities and adult schizophrenia-spectrum disorder. Schizophr Res 2006;81:253-60.

65 Schubert EW, Henriksson KM, McNeil TF. A prospective study of offspring of women with psychosis: visual dysfunction in early childhood predicts schizophrenia-spectrum disorders in adulthood. Acta Psychiatr Scand 2005;112:385-93.

66 Viertiö S, Laitinen A, Perälä J, et al. Visual impairment in persons with psychotic disorder. Soc Psychiatry Psychiatr Epidemiol 2007;42:902-8

67 Torrey EF, Bartko JJ, Yolken RH. Toxoplasma gondii and other risk factors for schizophrenia: an update. Schizophr Bull 2012;38:642-7.

68 Brown AS. Prenatal infection as a risk factor for schizophrenia. Schizophr Bull 2006;32:200-2.

69 Jeste DV, Gladsjo JA, Lindamer LA, et al. Medical comorbidity in schizophrenia. Schizophr Bull 1996;22:413-30.

70 Goldman LS. Medical illness in patients with schizophrenia. J Clin Psychiatry 1999;60:10-15.

71 Sokal J, Messias E, Dickerson FB, et al. Comorbidity of medical illnesses among adults with serious mental illness who are receiving community psychiatric services. J Nerv Ment Dis 2004;192:421-7. 